\title{
Relaciones entre hábitos de uso de videojuegos, control parental y rendimiento escolar
}

\author{
Daniel Lloret Irles, Víctor Cabrera Perona y Yolanda Sanz Baños \\ Universidad Miguel Hernández (España)
}

\begin{abstract}
Los menores dedican una media de 1.3 horas diarias a los videojuegos y la prevalencia de juego problemático oscila entre el $2 \%$ y el $8 \%$. Altos niveles de intensidad y frecuencia de juego, están asociados a un menor rendimiento escolar. El domicilio es el lugar preferido de juego, y por ello el control parental es decisivo. Objetivo: Analizar los patrones de juego e identificar relaciones significativas entre el control parental y el rendimiento académico. Material y método: Participantes: 610 estudiantes de ESO, con una media de edad de 13.84 años $(D T=1.27)$ y rango 12-16 años. Para analizar frecuencia, intensidad de juego, rendimiento académico y control parental se elaboraron escalas ad hoc. Resultados: Menores cuyos padres controlan el tiempo de juego y se interesan por los contenidos de forma continua, juegan significativamente menos días, menos horas y con contenidos ajustados a su edad. Aquellos con control parental discontinuo ("a veces"), aumentan la frecuencia e intensidad de juego, así como la proporción de contenidos no ajustados, y no se diferencian de los sin control parental. Además, un mayor número de horas de juego se relaciona con un menor rendimiento académico. Discusión/ conclusiones: Se avanza en la definición de la conducta problemática de juego. Los resultados indican que para que el control parental resulte efectivo éste debe ser continuo y consistente.
\end{abstract}

Palabras clave: Videojuegos, juego abusivo, rendimiento escolar, control parental.

Relationships between video-gaming habits, parental monitoring and school performance. Children spend an average of 1.3 hours per day on video game and problematic video gaming prevalence is set between $2 \%$ and $8 \%$. High levels of intensity and frequency of video gaming are associated with lower school achievement. Home is the most preferred place to play. Therefore parental monitoring is crucial. Objective: To analyse gaming patterns and to identify significant relationships between parental monitoring and academic performance. Method: Participants: 610 students of Secondary Education mean age 13.84 years $(S D=1.27$; range $12-16)$. Ad hoc scales were developed to analyse gaming frequency and intensity, school performance and parental monitoring. Results: Children, whose parents control gaming time and show interest in the contents continuously, play significantly fewer days, fewer hours and with adjusted contents to their age. Those with discontinuous parental control ("sometimes"), show a higher gaming frequency and intensity, and the proportion of unadjusted content, and do not differ from those without parental control. In addition, a greater number of hours of play are related to lower academic achievement. Discussion/ conclusions: This work helps in defining gambling problem behaviour. Results indicate that parental control is effective, on condition that it must be continuous and consistent.

Key words: Video game, school performance, problematic gaming, parental monitoring.

Correspondencia: Daniel Lloret. Facultad de Medicina. Universidad Miguel Hernández. Ctra. de Valencia, s/n. C.P. 03550. San Juan, Alicante (España). E-mail: daniel.lloret@umh.es 
Los videojuegos suponen para la mayoría de los jóvenes una estimulante actividad a la que dedican parte de su tiempo libre con el único objetivo de divertirse. Sin embargo, para una minoría, el juego se convierte en la actividad principal, el tiempo de juego aumenta a costa de reducir o incluso abandonar otras actividades placenteras y desatender sus responsabilidades. En casos extremos, la conducta de juego se vuelve compulsiva y se asocia con problemas sociales y/o emocionales. Su extendida penetración en los hogares españoles -Ferrer-López y Ruiz-San Román (2006) calculan que el $86.2 \%$ de los menores informan tener videojuegos en casa y el $90.8 \%$ declara haber jugado alguna vez- y la intensidad del uso ha suscitado una lógica preocupación sobre sus posibles efectos en el desarrollo cognitivo y social de los menores, y en consecuencia ha impulsado la investigación durante la última década. Los estudios epidemiológicos establecen la prevalencia de jugadores abusivos entre el 2 y el $10 \%$ (Ferrer-López y Ruiz-San Román, 2006; Gentile, 2009; Griffiths y Hunt, 1995; Lemmens, Valkenburg y Peter 2009; Mentzoni, Brunborg, Molde, Myrseth, Skouverøe, Hetland y Pallesen, 2011; Peukert, Sieslack, Barth y Batra, 2010; Rehbein, Kleimann y Möble, 2010; Sharif y Sargent, 2006; Van Rooij, Schoenmakers, Vermulst, Van Den Eijnden y Van De Mheen, 2011; Wenzel, Bakken y Johansson, 2009).

El uso de videojuegos se inicia en edades tempranas, cuando el menor se encuentra en proceso de socialización, influido por cuatro principales agentes: la familia, los amigos, la escuela y los medios de comunicación. De estos agentes, la familia destaca por su particular ascendencia en la adquisición de nuevas conductas y en la formación de actitudes en los menores (Bricker, Peterson, Sarason, Andersen y Rajan, 2007). Es por ello que consideramos que el control parental es decisivo en la regulación de la conducta de juego. La definición de control parental (parental monitoring o parental mediation) varía entre estudios. Algunos autores (Nathanson, 1999; Shin y Huh, 2011; Valkenburg, Krcmar, Peeters y Marseille, 1999; van der Voort, Nikken y van Lil, 1992) proponen tres estrategias de regulación parental: a) activa, b) restrictiva y c) el couso. La mediación activa consiste en promover la comunicación y tratar sobre aspectos relativos al contenido. La mediación restrictiva se basa en el establecimiento de normas que restringen el uso del medio, incluidas las restricciones relativas al tiempo y al contenido. El co-uso, radica en participar en la experiencia, pero sin hacer comentarios sobre el contenido o sus efectos. Sharif y Sargent (2006), sostienen que el control parental ejercería una influencia decisiva sobre el tiempo y tipo de juego. Sin embargo, no siempre es ejercido, y a menudo se emplea de forma inconsistente. Por lo general, los padres, en contra de lo recomendado por los especialistas en educación, apenas se implican en los juegos de sus hijos (ADESE, 2010; INTECO, 2011; Walsh, 2000). Es frecuente que sean los propios menores los que adquieran sus videojuegos y que jueguen a juegos con alto contenido violento y/o sexista (Ferrer-López y Ruíz-San Román, 2006).

En referencia a los efectos de los videojuegos sobre el rendimiento escolar, la evidencia sugiere que el uso problemático de videojuegos mantiene una relación inversa 
con el rendimiento escolar (Anand, 2007; Anderson et al., 2007; Gentile, Lynch, Linder y Walsh, 2004; Rehbein et al., 2010; Roe y Muijs, 1998), llegando incluso al desplazamiento del tiempo de estudio y al absentismo (Bartholow, Sestir y Davis, 2005; Gentile et al., 2004). Los escasos estudios longitudinales que abordan esta cuestión (Gentile et al., 2011; Lemmens, Valkenburg y Peter, 2011) encuentran una asociación entre el uso problemático de videojuegos y bajo rendimiento escolar. Si bien, niveles moderados de juego no se asocian con un bajo rendimiento escolar (Ferguson, 2011; León-Jariego y López, 2003; Skoric, Ching y Lijie, 2009). Incluso, podrían relacionarse con un mejor rendimiento escolar (Llorca, Bueno, Villar y Díez, 2010), una mejor habilidad visuoespacial (Boot, Kramer, Simons, Fabiani y Gratton, 2008), una mayor capacidad de atención (Shwan y Bavelier, 2003) y mejor coordinación motriz (Griffith, Voloschin, Gibb y Bailey, 1983). También McFarlane et al., (2002) describen las opiniones de profesores acerca de las bondades del juego moderado en sus alumnos, en cuanto a memorización, resolución de problemas y razonamiento deductivo.

A la vista de los resultados, podría afirmarse que el juego moderado resulta beneficioso y el abusivo perjudicial. Aunque la definición de juego abusivo o problemático aún está por concretar, lo que también afecta a los límites de la definición de juego moderado, parece demostrado que altos niveles de frecuencia e intensidad se asocian a un peor rendimiento escolar.

Teniendo en cuenta lo anterior, el presente trabajo pretende arrojar luz sobre la relación entre el control parental, la conducta de juego y el rendimiento escolar. A tal fin, tiene como objetivo identificar relaciones significativas entre control parental y perfil de juego, y su asociación con el rendimiento escolar. Nuestra primera hipótesis predice que los menores cuyos padres no se implican en la elección de los juegos jugarán en mayor medida a juegos no recomendados para su edad (Hipótesis 1). En relación a la frecuencia e intensidad de juego, esperamos que sea inversamente proporcional al control parental sobre el tiempo de juego. Es decir, a menor control parental, mayor frecuencia e intensidad de juego (Hipótesis 2). Asimismo, esperamos encontrar que los menores cuyos padres no controlan el tiempo de juego jugarán en mayor medida a juegos no recomendados a su edad (Hipótesis 3). Por último, en cuanto a la relación entre perfil de juego y rendimiento escolar, esperamos encontrar diferencia de medias en su rendimiento entre perfiles de jugadores (Hipótesis 4).

\section{MATERIAL Y MÉTODO}

\section{Participantes}

La muestra estuvo compuesta por 610 estudiantes de la ESO de 12 a 16 años $(M=13.84 ; D T=1.27)$, de la provincia de Alicante ( $51.5 \%$ chicos; $48.5 \%$ chicas). En las tablas 1 y 2 se detallan las puntuaciones obtenidas en las tres variables de estudio: 
rendimiento académico, control parental y perfil de juego. En relación a la primera, cabe resaltar que el $12.8 \%$ informa haber suspendido el último curso y el $10 \%$ una calificación de sobresaliente. El 22.6\% son repetidores. En cuanto al control parental, un $23.5 \%$ de la muestra declara que sus padres marcan un tiempo límite de juego, frente a un $22.2 \%$ que nunca lo hace. De la misma forma, un $33.5 \%$ refiere que sus padres se interesan por conocer los contenidos de los videojuegos, frente a un $24.4 \%$ que nunca lo hace. Un $26.6 \%$ de la muestra declara jugar a juegos recomendados para edades superiores. Los videojuegos son elegidos por el propio menor en un $80.5 \%$. En cuanto al perfil de juego, la mayoría juega preferentemente los fines de semana: el $31.6 \%$ juega sólo el fin de semana, y el $22.8 \%$ juega uno o dos días entre semana y uno o dos en fin de semana. La intensidad, medida en horas a la semana, refleja que la mayoría $(58.7 \%)$ juega menos de 10 horas y resalta que el $4.4 \%$ lo hace entre 30 y 60 horas o más. El resto de características puede consultarse en las tablas 1 y 2 .

Tabla 1. Características sociodemográficas

\begin{tabular}{|c|c|c|}
\hline$N=610$ & Porcentaje & Frecuencia \\
\hline \multicolumn{3}{|l|}{ Sexo } \\
\hline Chico & 51.5 & 314 \\
\hline Chica & 48.5 & 296 \\
\hline \multicolumn{3}{|l|}{ Curso } \\
\hline $1^{\circ} \mathrm{ESO}$ & 23.9 & 146 \\
\hline $2^{\circ} \mathrm{ESO}$ & 30.5 & 186 \\
\hline $3^{\circ} \mathrm{ESO}$ & 19.3 & 118 \\
\hline $4^{\circ} \mathrm{ESO}$ & 26.2 & 160 \\
\hline \multicolumn{3}{|l|}{ Repetidor } \\
\hline No & 77.4 & 472 \\
\hline $\mathrm{Si}$ & 22.6 & 138 \\
\hline \multicolumn{3}{|l|}{ Nota media del curso anterior } \\
\hline Suspenso & 12.8 & 78 \\
\hline Aprobado & 15.8 & 96 \\
\hline Bien & 26.6 & 162 \\
\hline Notable & 33.9 & 206 \\
\hline Sobresaliente & 10.0 & 61 \\
\hline \multicolumn{3}{|l|}{ Estado civil de los padres } \\
\hline Viven juntos & 74.9 & 457 \\
\hline Separados/divorciados & 21.0 & 128 \\
\hline Viudo/a & 2.3 & 14 \\
\hline Solteros & 1.1 & 7 \\
\hline $\mathrm{NS} / \mathrm{NC}$ & 0.4 & 4 \\
\hline \multicolumn{3}{|l|}{ Relación entre los padres } \\
\hline Muy buena & 54.3 & 326 \\
\hline Buena & 27.7 & 166 \\
\hline Regular & 9.2 & 55 \\
\hline Mal & 2.7 & 16 \\
\hline Muy mal & 1.3 & 8 \\
\hline No tienen trato & 4.8 & 29 \\
\hline \multicolumn{3}{|l|}{ Vive con } \\
\hline Padres & 77.7 & 475 \\
\hline Sólo madre & 17.8 & 108 \\
\hline Sólo padre & 2.3 & 14 \\
\hline Con ninguno de los dos & 0.8 & 5 \\
\hline Abuelos & 1.2 & 7 \\
\hline
\end{tabular}


Tabla 2. Características y hábitos de juego

\begin{tabular}{|c|c|c|}
\hline$N=610$ & Porcentaje & Frecuencia \\
\hline \multicolumn{3}{|l|}{ Ha jugado a videojuegos alguna vez } \\
\hline No & 2.0 & 12 \\
\hline $\mathrm{Si}$ & 98.0 & 598 \\
\hline \multicolumn{3}{|l|}{ Frecuencia de juego (Días-Semana) } \\
\hline No juega & 22.5 & 137 \\
\hline a) Sólo Fin de semana & 31.6 & 193 \\
\hline b) 1-2 días entre semana & 2.1 & 13 \\
\hline c) 3-4 días entre semana & 0.8 & 5 \\
\hline d) 1-2 días entre semana y 1-2 fin semana & 22.8 & 139 \\
\hline e) 3-4 días entre semana y 1-2 fin semana & 10.5 & 64 \\
\hline f) Todos los días & 9.7 & 59 \\
\hline \multicolumn{3}{|l|}{ Intensidad de juego (Horas semana) } \\
\hline No juega & 17.2 & 105 \\
\hline Juega semanalmente menos de $10 \mathrm{~h}$ & 58.7 & 358 \\
\hline Juega semanalmente entre $10 \mathrm{~h}-15 \mathrm{~h}$ & 13.8 & 84 \\
\hline Juega semanalmente entre $16 \mathrm{~h}-29 \mathrm{~h}$ & 4.9 & 30 \\
\hline Juega semanalmente 30h o más & 4.4 & 27 \\
\hline \multicolumn{3}{|c|}{ Ajuste (Juega a videojuegos recomendados para mayores) } \\
\hline No & 26.6 & 160 \\
\hline Sí & 37.9 & 228 \\
\hline No se fija en las recomendaciones & 36.6 & 214 \\
\hline \multicolumn{3}{|c|}{ Control parental tiempo (¿Padres marcan el tiempo de juego?) } \\
\hline No, nunca & 22.2 & 134 \\
\hline Sí, siempre & 23.5 & 142 \\
\hline A veces & 49.6 & 299 \\
\hline Sí, pero hago lo que quiero & 4.6 & 28 \\
\hline \multicolumn{3}{|c|}{ Control parental contenido (¿Padres se interesan por conocer sus juegos?) } \\
\hline No, nunca & 24.4 & 147 \\
\hline Sí, siempre & 33.5 & 202 \\
\hline A veces & 43.2 & 261 \\
\hline
\end{tabular}

\section{Procedimiento}

Tres centros educativos fueron seleccionados mediante un muestreo no probabilístico (incidental) y estratificado. La muestra fue seleccionada en función de la representatividad geográfica y social (Centro público: 43\%, Privado: 57\%; nivel socioeconómico variable). Tras la aprobación del proyecto por parte del Consejo Escolar y Claustro de los centros educativos, se acordaron las fechas de administración. Una vez informadas las AMPAS del objetivo, procedimiento y metodología del estudio, se obtuvieron los consentimientos firmados. La recogida de datos se realizó en tres meses. El procedimiento fue autoadministrado y el tiempo de cumplimentación osciló entre 30 y 40 minutos. Antes de administrar los cuestionarios, se explicaba al grupo detalladamente la mecánica de cumplimentación y se garantizaba el anonimato.

\section{Variables e instrumentos}

Cuestionario Sociodemográfico y de rendimiento académico elaborado ad hoc. Cuestionario autoaplicado, compuesto por 14 ítems con distintos formatos de respuesta: cerrada, dicotómica y/o escala tipo Likert de 5 opciones de respuesta.

Cuestionario de hábitos de uso de videojuegos y nuevas tecnologías elaborado ad hoc, a partir de los hallazgos de investigaciones previas, compuesto por 20 
ítems. Los formatos de respuesta consisten en preguntas cerradas con respuesta dicotómica, respuesta múltiple y tipo Likert de cinco opciones.

La conducta de juego quedó definida por tres dimensiones: ajuste, frecuencia e intensidad. El ajuste hace referencia a que el menor juega a juegos recomendados para su edad. La frecuencia indica el número de días que juega a la semana. Se distingue entre días laborales y fines de semana, produciéndose 6 categorías de jugadores: a) Sólo fin de semana; b) Perfil bajo entre semana (juegan 1 ó 2 días entre semana); c) Perfil alto entre semana (juegan 3 ó 4 días entre semana); d) Perfil bajo diario (juegan 1 ó 2 días entre semana +1 ó 2 días en fín de semana); e) Perfil alto diario (juegan 3 ó 4 días entre semana +1 ó 2 días en fin de semana) y f) Juegan todos los días. En cuanto la intensidad, hace referencia al número de horas de juego a la semana. La variable se distribuye en 4 categorías: a) Juega menos de 10 horas; b) Juega de 10 a 15 horas; c) Juega de 15 a 29 horas; d) Juega 30 o más horas.

\section{Tratamiento de datos}

Para la codificación y análisis de los datos se utilizó el programa estadístico SPSS 18.0. Se han realizado análisis descriptivos de tendencia central reflejándose los porcentajes, las medias y las desviaciones típicas. Se estudiaron correlaciones entre las variables, utilizando coeficientes de correlación de Pearson. Para el análisis de variables cualitativas se utilizaron tablas de contingencia de doble entrada y se calculó Chi-cuadrado, a un nivel de confianza del 95\%. También se realizaron análisis de diferencia de medias para muestras independientes (prueba T-Student).

\section{RESULTADOS}

\section{Control parental sobre los contenidos}

En relación al ajuste con la edad, se observaron diferencias significativas entre los menores cuyos padres ejercen control parental por los contenidos de los juegos, y los que no $\left(\chi^{2}=73.933 ; p<.001\right)$. Cuando los padres se interesan por los contenidos, el $55.7 \%$ no juegan a juegos recomendados para mayores. Sin embargo, cuando no se interesan el porcentaje se reduce al $15.8 \%$. Y si el interés de los padres es inconsistente ("a veces"), sólo la cuarta parte afirma no jugar a juegos para mayores.

Se encontraron diferencias significativas entre la frecuencia $\left(\chi^{2}=40.496\right.$; $p<.001)$ e intensidad $\left(\chi^{2}=75.853 ; p<.001\right)$ y el control parental por los contenidos de los juegos (ver tabla 3). Cabe reseñar que las correlaciones entre las escalas de frecuencia e intensidad se mostraron significativas, con valor superior a .068 ( $p<.001)$. Se observa que valores de frecuencia más altos se asocian a valores de control parental más bajo: un $43.5 \%$ en los jugadores de fin de semana y un $17.2 \%$ en los jugadores de diario. En cuanto a intensidad, los jóvenes cuyos padres ejercen control parental consistente por el 
contenido de los juegos juegan en un $37.7 \%$ menos de 10 horas, frente a sólo un $3.8 \%$ que lo hace 30 horas o más. Estas diferencias confirman la primera hipótesis.

Tabla 3. Ajuste, frecuencia e intensidad en función del control parental por el contenido

\begin{tabular}{|c|c|c|c|c|c|}
\hline & \multicolumn{4}{|c|}{ ¿Tus padres se interesan por conocer tus juegos? } & \multirow{2}{*}{$\chi^{2}(p)$} \\
\hline & Siempre & Nunca & A veces & $\%$ Total & \\
\hline \multicolumn{5}{|l|}{ Ajuste } & \multirow{4}{*}{$73.933^{* *}$} \\
\hline Sí juego a juegos para mayores. & 25 & 30.7 & 44.3 & 100 & \\
\hline No juego a juegos para mayores. & 55.7 & 15.8 & 26.6 & 100 & \\
\hline No me fijo en las recomendaciones & 26.9 & 32.4 & 41.5 & 100 & \\
\hline \multicolumn{5}{|l|}{ Frecuencia } & \multirow{7}{*}{$40.496^{* *}$} \\
\hline Sólo fin de semana(a) & 43.5 & 15.5 & 40.9 & 100 & \\
\hline Perfil bajo entre semana(b) & 23.1 & 15.4 & 61.5 & 100 & \\
\hline Perfil alto entre semana(c) & 20 & 60 & 20 & 100 & \\
\hline Perfil bajo diario(d) & 23.9 & 24.6 & 51.4 & 100 & \\
\hline Perfil alto diario(e) & 23.4 & 35.9 & 40.6 & 100 & \\
\hline Juegan todos los días(f) & 17.2 & 41.4 & 41.4 & 100 & \\
\hline \multicolumn{5}{|l|}{ Intensidad } & \multirow{5}{*}{$75.853^{* *} *$} \\
\hline Juegan menos de 10 horas(a) & 37.7 & 18.2 & 44.1 & 100 & \\
\hline Juegan de 10 a 15 horas(b) & 16.9 & 38.6 & 44.6 & 100 & \\
\hline Juegan de 15 a 28 horas(c) & 30 & 40 & 30 & 100 & \\
\hline Juegan de 30 a 60 horas(d) & 3.8 & 38.5 & 57.7 & 100 & \\
\hline
\end{tabular}

\section{Control parental del tiempo de juego}

Con respecto a la relación entre el control parental del tiempo de juego y el ajuste de la edad recomendada del jugador $\left(\chi^{2}=32.794 ; p=.001\right)$, se encontró que $36.9 \%$ de los menores con control consistente no juegan a juegos para mayores. Cuando los padres no ejercen control, tan sólo un $15.9 \%$ de los menores no jugaban a juegos para mayores (ver tabla 4). Por lo tanto, los menores cuyos padres no controlan el tiempo de juego juegan en mayor medida a juegos no recomendados a su edad, tal y como predice la hipótesis 3.

Se encontraron diferencias significativas en la frecuencia de juego en función del control del tiempo por parte de los padres $\left(\chi^{2}=62.472 ; p<.001\right)$. La ausencia de control se relacionó con una mayor frecuencia: juego diario $(27.1 \%)$, perfil alto diario $(34.4 \%)$ y perfil alto entre semana $(40 \%)$. Por el contrario, un control consistente se relacionó con una menor frecuencia; jugadores sólo fin de semana (33.3\%) y perfil bajo semana $(23.1 \%)$.

En cuanto a la intensidad de juego, también se hallaron diferencias significativas $\left(\chi^{2}=45.226 ; p<.001\right)$. En los participantes cuyos padres controlan el tiempo de juego, los valores más altos se asocian a una menor frecuencia de juego. Por el contrario, cuando el control parental no existe, y los mayores valores se acumulan en altas frecuencias de juego. De esta forma, en jugadores de menos de 10 horas/semana el $28.5 \%$ tenía control parental, frente a un $17.6 \%$ sin control. En jugadores de 30 o más horas, los valores se invierten: $7.4 \%$ con control parental y $29.6 \%$ sin control (Tabla 4). 
La hipótesis 2, a menor control parental, mayor frecuencia e intensidad de juego, queda confirmada.

Tabla 4. Ajuste, frecuencia e intensidad en función del control parental del tiempo de juego

\begin{tabular}{|c|c|c|c|c|c|c|}
\hline & \multicolumn{4}{|c|}{ ¿Tus padres marcan el tiempo de juego? } & \multirow[b]{2}{*}{$\%$ Total } & \multirow[b]{2}{*}{$\chi^{2}(p)$} \\
\hline & Sí, siempre & No, nunca & A veces & $\begin{array}{l}\text { Sí, pero hago } \\
\text { lo que quiero }\end{array}$ & & \\
\hline \multicolumn{6}{|l|}{ Ajuste } & \multirow{4}{*}{$32.794 * *$} \\
\hline $\begin{array}{l}\text { Sí juego a juegos para mayores. } \\
\text {. }\end{array}$ & 18.4 & 25.4 & 50.9 & 4.4 & 100 & \\
\hline No juego a juegos para mayores. & 36.9 & 15.9 & 45.9 & 1.3 & 100 & \\
\hline No me fijo en las recomendaciones & 18.6 & 24.5 & 51 & 5.9 & 100 & \\
\hline \multicolumn{6}{|l|}{ Frecuencia } & \multirow{7}{*}{$62.472 * *$} \\
\hline Sólo fin de semana(a) & 33.2 & 14.0 & 50.8 & 2.1 & 100 & \\
\hline Perfil bajo entre semana(b) & 23.1 & 15.4 & 53.8 & 7.7 & 100 & \\
\hline Perfil alto entre semana(c) & - & 40.0 & 60.0 & - & 100 & \\
\hline Perfil bajo diario(d) & 21.7 & 20.3 & 54.3 & 2.9 & 100 & \\
\hline Perfil alto diario(e) & 7.8 & 34.4 & 53.1 & 3.1 & 100 & \\
\hline Juegan todos los días(f) & 10.2 & 27.1 & 47.5 & 15.3 & 100 & \\
\hline \multicolumn{6}{|l|}{ Intensidad } & \multirow{5}{*}{$45.226^{* *}$} \\
\hline Juegan menos de 10 horas(a) & 28.5 & 17.6 & 51.4 & 2.2 & 100 & \\
\hline Juegan de 10 a 15 horas(b) & 13.3 & 28.9 & 49.4 & 8.4 & 100 & \\
\hline Juegan de 15 a 28 horas(c) & 10 & 23.3 & 50 & 13.3 & 100 & \\
\hline Juegan de 30 horas o más (d) & 7.4 & 29.6 & 55.6 & 7.4 & 100 & \\
\hline
\end{tabular}

\section{Rendimiento académico}

Se realizaron pruebas de contraste de medias para analizar las diferencias en el rendimiento académico según la frecuencia y la intensidad de juego (ver tabla 5). En cuanto a la frecuencia de juego, se crearon dos grupos polarizados: Alto, compuesto por: Juegan perfil alto entre semana (3 ó 4 días) + juegan perfil alto diario (3 ó 4 entre semana + 1-2 fin de semana) + juegan todos los días; y Bajo: Juegan fin de semana + juegan perfil bajo entre semana (1 ó 2 días) + juegan perfil bajo diario (1 ó 2 entre semana +1 ó 2 fin de semana).

Las diferencias $(t=-2.449 ; p<.05)$, aunque pequeñas, están explicadas en un $11.21 \%$ por el perfil de frecuencia de juego Alto/Bajo. Estas diferencias son significativas. Los de bajo perfil obtienen una mejor nota media (situándose entre el notable y el bien), frente a la nota media de los de perfil alto que tiende hacia el bien/aprobado.

Atendiendo a la intensidad de juego, los grupos polarizados fueron: Alto: Juegan más de 10 horas semanales; y Bajo: Juegan menos de 10 horas semanales. Las diferencias fueron significativas $(t=-3.420 ; p<.005)$ y aunque pequeñas, están explicadas en un $\mathbf{1 5 . 1 6 \%}$ por la condición de menos o más de 10 horas de juego. Los que juegan menos de 10 horas obtienen una mejor nota media (situándose entre el notable y el bien), frente a la nota media de los que juegan más de 10 horas que tiende hacia el bien/aprobado. Por lo que se confirma la hipótesis 4. 
Tabla 5. Prueba-T con respecto al rendimiento académico

\begin{tabular}{cccccccc}
\hline \multicolumn{1}{c}{ Variables } & Grupo & $N$ & Media & $S D$ & $t$ & gl & $p$ \\
\hline \multirow{2}{*}{ Frecuencia de Juego } & Alta & 128 & 3.10 & 1.266 & \multirow{2}{*}{-2.441} & \multirow{2}{*}{471} & \multirow{2}{*}{$.015^{*}$} \\
\cline { 2 - 6 } & Baja & 345 & 2.81 & 1.112 & & & \\
\hline \multirow{2}{*}{ Intensidad de Juego } & Alta & 141 & 3.13 & 1.191 & \multirow{2}{*}{-3.420} & \multirow{2}{*}{497} & \multirow{2}{*}{$.001^{* *}$} \\
\cline { 2 - 6 } & Baja & 358 & 2.75 & 1.125 & & & \\
\hline$* * p<.001 ; * p<.05$ & & & & & & &
\end{tabular}

\section{CONCLUSIONES}

El objetivo del presente trabajo es determinar si existe relación entre el control parental y el uso problemático de videojuegos, y si este a su vez se relaciona con el rendimiento escolar.

Padres y educadores comparten su preocupación sobre en qué medida el uso excesivo de videojuegos afecta al rendimiento escolar. El rol predominante de las madres y los padres en la educación de la prole, hace del control parental una estrategia obligatoria para la temprana regulación de la conducta de juego, como base de un hábito saludable. Nuestros resultados muestran como el control parental mantiene una relación inversa con la edad del menor, de manera que disminuye al aumentar la edad. Estos datos son congruentes con otros estudios (Nikken y Jansz, 2006; Shin y Huh, 2011) y se justifican desde un enfoque educativo en el que el menor obtiene gradualmente su autonomía, partiendo de un control más restrictivo.

Nuestros resultados concluyen que un consistente control parental sobre el tiempo de juego y los contenidos se asocia con un perfil de jugador no problemático. Ya sea por no presentar tiempos excesivos de juego, como por elegir juegos recomendados para su edad. De esta manera, los menores cuyos padres muestran un interés constante por los contenidos y por el tiempo de juego, juegan significativamente menos a juegos de adultos. Observamos como un control parental constante sobre el tiempo de juego aumenta significativamente la asociación con jugar únicamente el fin de semana. Sin embargo, cuando el control parental es ocasional, la asociación con jugar a juegos de adultos aumenta y no se diferencia de los menores sin supervisión alguna. Por lo tanto, el control parental se muestra realmente efectivo cuando es percibido como una supervisión continua y consistente en el tiempo. Estos resultados verifican las tres primeras hipótesis planteadas.

Hay que decir aquí que el control parental no termina en la supervisión del tiempo y de los contenidos. Algunos autores consideran que la ubicación del dispositivo de juego es una variable importante (ADESE, 2010), y aconsejan no ubicarlo en la habitación del menor, sino en un espacio compartido, como el salón. Sin embargo, esta variable no ha sido considerada por la difusión del uso de dispositivos portátiles, que dificulta el control sobre el lugar de juego. Otra posible crítica, se refiere a haber recabado solamente información de los menores y no de los padres. Bovill y Livingstone 
(2001) muestran que una información mixta proveniente de ambas fuentes es mucho más fiable. Por su parte Nikken y Jansz (2006) señalan en su investigación que los menores informaron que tenían menos control que la información aportada por los padres.

En relación al rendimiento escolar y el perfil de juego, tanto los resultados relativos a la frecuencia, como los referidos a la intensidad confirman la hipótesis planteada. Hubo diferencias entre perfiles, pero no demasiado grandes, aunque sí significativas. Cabe aquí reflexionar sobre el hecho de que la conducta de juego se refiera al momento de la toma de datos, mientras que el rendimiento escolar se refiere a la nota del curso pasado. Es plausible pensar que la frecuencia e intensidad informadas pudieran no ser las mismas un año atrás cuando tuvo lugar la nota.

No obstante las limitaciones señaladas, creemos que el presente trabajo aporta información de utilidad en la definición de la conducta problemática de juego y sirve de soporte para el diseño de acciones en el ámbito de la prevención familiar.

\section{REFERENCIAS}

Asociación Española de Distribuidores y Editores de Software de Entretenimiento-ADESE (2010). Informe sobre usos y hábitos de los videojugadores españoles.

Anderson, C.A. et al. (2007). Violent video games effects on children and adolescents: theory, research, and public policy. Oxford: Oxford University Press.

Anand, V. (2007) A study of time management: the correlation between video game usage and academic performance markers. Cyberpsychology y Behavior: The Impact Of The Internet, Multimedia And Virtual Reality On Behavior And Society 10(4), 552-9.

Bartholow, B.D., Sestir, M.A. y Davis, E.B. (2005). Correlates and Consequences of Exposure to Video Game Violence: Hostile Personality, Empathy, and Aggressive Behavior. Personality and Social Psychology Bulletin, 31(11), 1573-1586.

Bovill, M. y Livingstone, S. (2001). Bedroom culture and the privatization of media use. In: Livingstone, S. y Bovill, M. (Eds). Children and their changing media environment: A European comparative study. Mahwah, NJ: Lawrence Erlbaum, 179-200.

Boot, W.R., Kramer, A.F., Simons, D.J., Fabiani, M. y Gratton, G. (2008). The effects of video game playing on attention, memory, and executive control. Acta Psychologica, 129(3), 387-398

Bricker, J.B., Peterson, A.V., Sarason, I.G., Andersen, M.R. y Rajan, K.B. (2007). Changes in the influence of parents' and close friends' smoking on adolescent smoking transitions. Addictive Behaviours, 32, 740-757.

Ferguson, C.J. (2011). The influence of television and video game use on attention and school problems: A multivariate analysis with other risk factors controlled. Journal of Psychiatric Research, 45(6), 808-813.

Ferrer-López, M. y Ruiz, J.A. (2006). Uso de videojuegos en niños de 7 a 12 años Una aproximación mediante encuesta. Icono, 14, 7.

Gentile, D.A. (2009). Pathological Video-Game Use Among Youth Ages 8 to 18. Psychological Science, 20(5), 594-602.

Gentile, D.A., Lynch, P.J., Linder, J.R. y Walsh, D.A. (2004 ). The Effects of Violent Video Game Habits on Adolescent Hostility, Aggressive Behaviors, and School Performance. Journal of Adolescence, 27, 5-22. 
Gentile, D.A., Choo, H., Liau, A., Sim, T., Li, D., Fung, D., et al. (2011). Pathological Video Game Use Among Youths: A Two-Year Longitudinal Study. Pediatrics, 127(2), 319329.

Griffith, J.L., Voloschin, P., Gibb, G.D. y Bailey, J.R. (1983). Differences in eye-hand motor coordination of video-game users and non-users. Percept Mot Skills, 57(1), 155-158.

Griffiths, M.D. y Hunt, N. (1995) Computer gaming playing in adolescent: Prevalence and demographic indicators. Journal of Community and Applied Social Psychology, 5(3), 189-193.

INTECO (2012). Los controles parentales: cómo vigilar a qué contenidos de Internet acceden nuestros hijos.

Lemmens, J.S., Valkenburg, P.M. y Peter, J. (2009). Development and validation of a game addiction scale for adolescents. Media Psychology, 12, 77-95.

Lemmens, J.S., Valkenburg, P.M. y Peter, J. (2011). Psychosocial causes and consequences of pathological gaming. Computers in Human Behavior, 27(1), 144-152.

León, R. y López, M.J. (2003). Los adolescentes y los videojuegos. Apuntes de Psicología, 21(1), 89-99.

Llorca, M.A., Bueno, G.M., Villar, C. y Díez, M.A. (2010). Frecuencia en el uso de videojuegos y rendimiento académico. II Congreso Internacional Comunicación 3.0. Salamanca.

McFarlane, A., Parrowhawk, A. y Heald, Y. (2002). Report on the educational use of games. Disponible en www.teem.org.uk/publications/teem_gamesined_full.pdf. Recuperado el 4 Febrero, 2012.

Mentzoni, R.A., Brunborg, G.S., Molde, H., Myrseth, H., Skouverøe, K.J., Hetland, J. y Pallesen, S. (2011). Problematic Video Game Use: Estimated Prevalence and Associations with Mental and Physical Health. Cyberpsychology, Behavior, and Social Networking, 12(2), DOI: $10.1089 /$ cyber.2010.0260

Nathanson, A.I. (1999). Identifying and explaining the relationship between parental mediation and children's aggression. Communication Research, 26(2), 124-143.

Nikken, P. y Jansz, J. (2006). Parental mediation of children's videogame playing: a comparison of the reports by parents and children. Learning, media and technology, 31(2), 181-202.

Peukert, P., Sieslack, S., Barth, G. y Batra, A. (2010) Internet and computer game addiction: phenomenology, comorbidity, etiology, diagnostics and therapeutic implications for the addictives and their relatives. Psychiatric Praxis, 37, 219-224.

Rehbein, F., Kleimann, M. y Mößle, T. (2010) Prevalence and risk factors of video game dependency in adolescence: Results of a German nationwide survey. Cyberpsychology, Behavior and Social Networking, 13(3), 269-277.

Roe, K. y Muijs, D. (1998). Children and Computer Games. A Profile of Heavy User. European Journal of Communication, 13(2), 181-200.

Sharif, I. y Sargent., J.D. (2006) Association between television, movie, and video game exposure and school performance. Pediatrics, 118(4), 61-70.

Shwan C. y Bavelier D. (2003) Action video game modifies visual selective attention. Nature, 423, 534-537.

Shin, W. y Huh, J. (2011). Parental mediation of teenagers'video game playing: Antecedents and consequences. New Media y Society, 13, 945-962.

Skoric, M.T., Ching Neo, L. y Lijie, R. (2009). Children and video games: Addiction, engagement, and scholastic achievement. CyberPsychology and Behavior, 12(5), 567-572.

Valkenburg, P.M., Krcmar, M., Peeters, A.L. y Marseille, N.M. (1999). Developing a scale to assess three different styles of television mediation: "instructive mediation," "restrictive mediation," and "social coviewing". Journal of Broadcasting y Electronic Media, 43, 52 66. 
Van der Voort, T.H.A., Nikken, P. y van Lil, J.E. (1992). Determinants of parental guidance of children's television viewing: A Dutch replication study. Journal of Broadcasting $y$ Electronic Media, 36, 61-74.

Van Rooij, A.J., Schoenmakers, T.M., Vermulst, A.A., Van Den Eijnden, R.J.J.M. y Van De Mheen, D. (2011). Online video game addiction: Identification of addicted adolescent gamers. Addiction, 106, 205-212.

Walsh, D.A. (2000). Interactive violence and children: testimony submitted to the Committee on Commerce, Science, and Technology, United States Senate. Minneapolis, MN: National Institute on Media and the Family. Disponible en: http://www.mediafamily.org/press/senateviolence-full.shtml. Recuperado el 10 Enero, 2013.

Wenzel, H.G, Bakken I.J, Johansson, A., Götestam K.G. y Øren, A. (2009) Excessive computer game playing among Norwegian adults: self-reported consequences of playing and association with mental health problems. Psychologic Report, 105, 1237-1247.

Recibido: 21 de febrero de 2013

Recepción Modificaciones: 8 de marzo de 2013

Aceptado: 22 de agosto de 2013 